\section{Eye Pathology Scheme}

\author{
I. H. CHISHOLM and J. MARSHALL \\ London
}

The aim of the scheme is to assist interested clinicians and research workers in obtaining pathological material from the donor eyes of patients previously identified as having disorders requiring further investigation. It is modelled on that already organised by the British Retinitis Pigmentosa Society (BRPS) and is dependent on the co-operation and advice of the United Kingdom Transplant Service (UKTS) and the Institute of Ophthalmology, University of London.

The BRPS scheme was initiated six years ago and now has 500 registered donors. Between Donder's first description of retinitis pigmentosa (RP) in the 1850 s and 1980 only about 40 globes from RP sufferers had been subjected to laboratory study and almost all of these were used solely for histopathology. In the last 6 years under the BRPS scheme more than 50 eyes have been made available. This has resulted in a far better understanding of the ultrastructural changes occurring in RP and has enabled a number of dynamic studies to be undertaken on cells in tissue culture. Cell lines have been established from different types of RP and have been exchanged between laboratories in this country and elsewhere. Biochemical studies have also been undertaken to try to establish the underlying abnormality involved. It is now proposed to establish a similar scheme for other forms of eye disease.

\section{Outline of the scheme}

The scheme is open to medical practitioners and those working under their supervision. The purposes for which eyes might be requested include clinico-pathological correlation, tissue culture, anatomical survey, biochemical analysis or other specific reasons to meet individual needs. The request may form part of an individual's research investigation or of a wider collaborative project.

The clinician will, following discussion with the patient, obtain the appropriate informed consent and complete the registration details returning these to the scheme office. The UKTS will act as a clearing house informing the registering clinician as and when the donated eyes become available. The clinician will arrange for the collection of the eyes unless alternative arrangements have been made. Any costs involved will, however, be the responsibility of the requesting unit. Any necessary ethical approval must be obtained by the requesting clinician.

\section{Registration Particulars}

Registration forms, which include an appropriate consent form, are available from the scheme office at the Institute of Ophthalmology, Cayton Street, London EC1V 9AT (Fig. 1). These no carbon copy forms should be completed and returned to the scheme office. The registration particulars will be recorded, registered with the UKTS, and a copy returned to the registering clinician together with a copy of the consent. The second copy of the consent form will be sent to the patient's general practitioner together with a covering letter. The patient will be thanked for his co-operation and will be issued with a membership card indicating his part in the scheme. Other clinicians who are involved will be notified when appropriate. 


\section{EYE PATHOLOGY SCHEME}

Requesting clinician

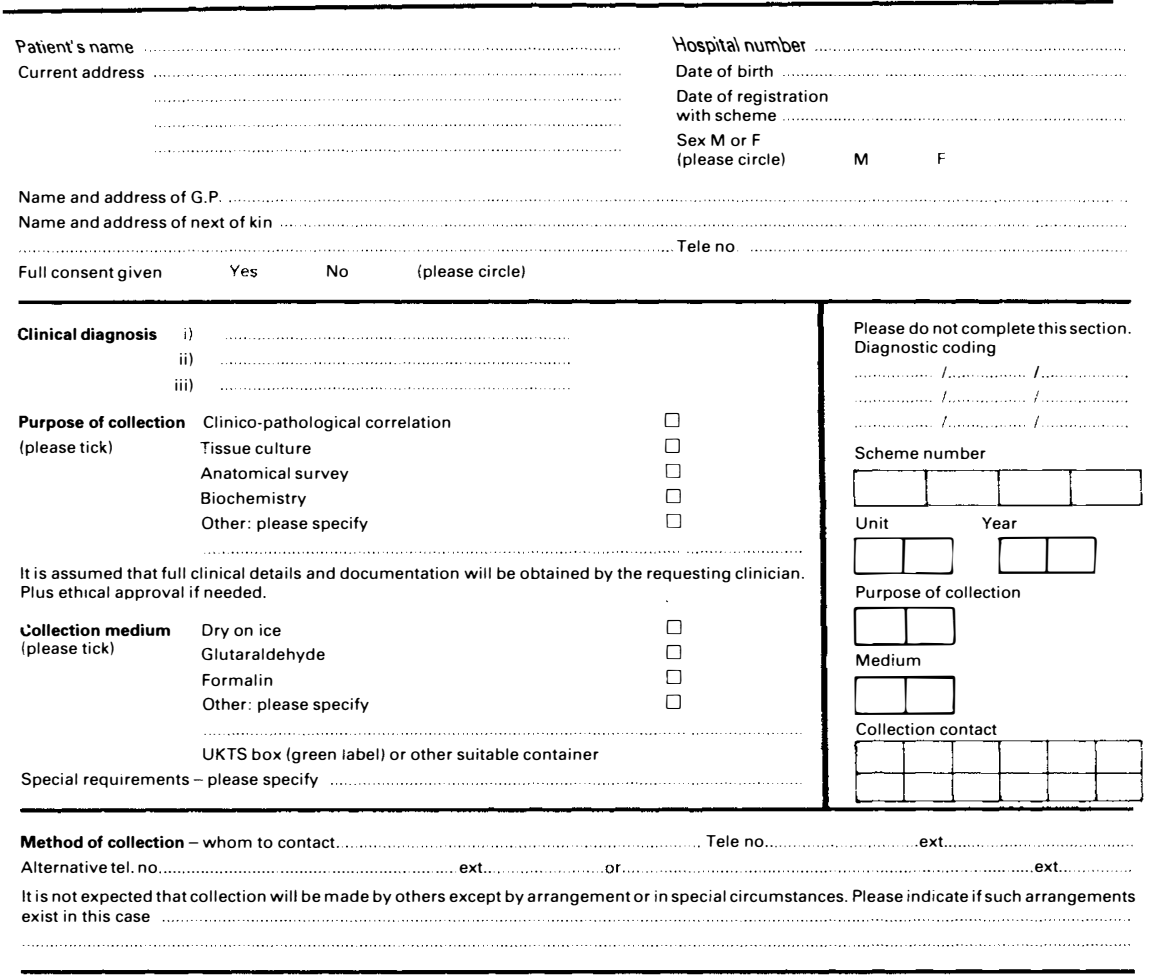

Please return to:

Mr. I. H. Chisholm, FRCS, Institute of Ophthalmology, Cayton Street, London EC1V 9AT

hereby consent to the donation of my eyes in the event of my death forthe purpose of further study into the underlying basis of my eye problem.

Signed

Next of kin

Address

General Practitioner Dr

Address

Ophthalmologist $\mathrm{Mr}$

Address

Please return the 3 copies of the completed form to:

Mr. I. H. Chisholm, FRCS, Institute of Ophthalmology, Cayton Street, London EC1V 9AT 


\section{Tissue collection}

In the event of the patient's death the UK Transplant Service should be informed on Bristol 0272507777 by the patient's relatives, general practitioner or clinician involved. It should be stressed that the eyes are being collected under the Eye Pathology Scheme and not for transplant purposes. The importance of this will be emphasised in all the documentation sent to those concerned. There will also be checks made in Bristol to avoid material going astray. Should the eyes be collected locally without going through the UKTS it is still requested that 'UKTS be informed so as to remove the patient's name from file. This will avoid further checks being made at a later date either with the patient's family or the registering physician.

When the UKTS is informed that eyes are available the registering clinician will be contacted to that effect. If no contact is made with him then the alternative contact indicated on the registration form will be informed. It will be for the clinician to arrange to have the eyes collected unless other arrangements have been made. Information as to the storage medium are included on the form to ensure that the eye is properly stored for transport. The UKTS will, if required, provide suitable collection boxes which will have a distinctive label identifying that the eyes are not for transplantation purposes. Alternatively any suitable container may be used. The collection fluids needed should be provided locally unless of a specialised nature in which case the requesting clinician will have to help as necessary.

Should the patient die outside the area of the requesting clinician or it be impossible for the clinician to arrange to collect the eyes then the UKTS will try to assist in contacting a unit nearer the patient. Several university units have assisted in the collection of eyes under the BRPS scheme and it is hoped that a similar facility will be available and that consultants using the scheme will recover material for others should it become available in their area. This will depend on the goodwill of all concerned. The UKTS will offer assistance, as with all donor tissue, in transporting eyes between units when necessary. Equally when groups of ophthalmologists or research work- ers are involved in a project relating to donor material UKTS will assist in getting the eyes to the appropriate destination with a minimum of delay as required by the nature of the material.

The pathological processing and further investigation of the material obtained will be the responsibility of the requesting clinician in conjunction with appropriate pathologists and others. The co-ordination of this and the responsibility for the costs involved fall outside the scheme which aims to facilitate the obtaining of appropriate material rather than to provide a pathology service.

\section{Cost and administration of the scheme}

There will be no charge for the registration and notification aspects of the scheme but when additional collection and transport costs are incurred then these will have to be borne by the requesting clinician. This also applies to the costs incurred by any other unit on his behalf.

The scheme will be administered from the Institute of Ophthalmology, Cayton Street, London EC1V 9AT to which all correspondence and registration forms should be sent. Any queries should be addressed to $\mathrm{Mr}$ I. H. Chisholm either at the Institute or Southampton Eye Hospital, Wilton Avenue, Southampton SO9 $4 \mathrm{XW}$. The original records will be held at the Institute and the necessary details passed to UKTS. The data will be stored on computer and be covered under the Data Protection Act. The scheme has the approval and support of the Faculty of Ophthalmologists.

\section{Development of the scheme}

The purpose of the Eye Pathology Scheme is to provide a central register of patients whose eyes have been requested and donated for pathological research after their death. The scheme should also be able to play a role in co-ordinated research but this should not in any way interefere with the investigation and research carried out by any clinician or pathologist on his own. In setting up the scheme it is, however, hoped that a greater interest can be stimulated into the investigation of ocular 
pathology either by an individual or a group of interested people.

It is anticipated that as the scheme develops periodic checks will be made both to confirm the patient's current address and the clini cian's continuing need for the eyes should they become available. In keeping contact with the patient all will be aware of the implications of the scheme and the informed consent already given. 